\title{
Prevention of biliary stent occlusion using cyclical antibiotics and ursodeoxycholic acid
}

\author{
S Ghosh, K R Palmer
}

\begin{abstract}
This study reports an open randomised controlled trial of cyclical antibiotics and ursodeoxycholic acid in prevention of plastic biliary stent occlusion. Seventy patients with malignant distal bile duct obstruction were randomised to either active treatment with cyclical antibiotics (ampicillin, metronidazole, ciprofloxacin) and ursodeoxycholic acid or no treatment after successful stent insertion. The two groups were well matched. The follow up was complete with stent occlusion or death being the end points. There was no difference in the incidence of stent occlusion between the two groups and the overall survival was similar. In conclusion, this study did not show any benefit of treatment with antibiotics and ursodeoxycholic acid in prolonging stent patency or improving survival.
\end{abstract}

(Gut 1994; 35: 1757-1759)

Endoscopically placed stents are widely used to relieve malignant extrahepatic obstructive jaundice. Unfortunately, plastic stents may block and this leads to jaundice and cholangitis. It is possible that stent occlusion occurs as a consequence of low biliary flow and bacterial adhesion. ${ }^{12}$ We reasoned that a combination of systemic antibiotics and a choleretic agent could reduce the rate of stent occlusion.

In this study we have examined the effects of cyclical antibiotics and oral ursodeoxycholic acid treatment upon stent occlusion rates. We used cyclical antibiotics, which are well excreted into bile to reduce the likelihood of producing antibiotic resistant bacterial strains in bile. Ursodeoxycholic acid is modestly choleretic and its use may be helpful in a range of cholestatic liver diseases. ${ }^{3}$

\section{Methods}

\section{PATIENTS}

Eighty seven patients ( 52 men) aged 62 to 85 (median 70) years were recruited between January 1991 and October 1992. All presented with extrahepatic obstructive jaundice; 62 patients had pancreatic cancer and 25 had cholangiocarcinoma or secondary tumours occluding the bile duct. Diagnosis was made on clinical grounds, ultrasonography or computed tomography imaging, by typical appearances at endoscopic retrograde cholangiopancreatography (ERCP) and by progression of disease. Tissue confirmation of the diagnosis was made in only eight cases.
All patients had a serum bilirubin of at least $50 \mu \mathrm{mol} / 1$ and had distal common bile duct strictures (within $4 \mathrm{~cm}$ of the ampulla of Vater). All had irresectable tumours based upon factors such as age, their general medical condition, and ultrasound or computed tomography evidence of hepatic metastasis or vascular invasion. Patients with resectable tumours, proximal bile duct or bifurcation tumours, those with endoscopic evidence of duodenal stenosis or previous gastric surgery in whom endoscopic stenting was impossible were not considered. In addition, patients whose general condition suggested a very short survival were excluded from the study.

\section{DESIGN}

Biliary stent insertion was carried out at ERCP (combined with percutaneous access in three patients) under cover of prophylactic antibiotics (IV ampicillin and gentamicin or piperacillin). The stents were $10 \mathrm{~F}-12 \mathrm{~F}$ in diameter with end holes and 8-14 cm long. The patients received IV piperacillin $2 \mathrm{~g}$ thrice daily for 48 hours after stent insertion. After stent insertion liver function tests were repeated to ensure a successful functional result. If the serum bilirubin had fallen by at least $20 \%$ within 14 days the patients were randomised to active treatment or no treatment. Active treatment consisted of both: (a) continuous treatment with ursodeoxycholic acid $10 \mathrm{mg} / \mathrm{kg} /$ day given as a single night time dose; (b) monthly cycles of three antibiotics: (a) ampicillin $500 \mathrm{mg}$ four times daily; (b) metronidazole $400 \mathrm{mg}$ twice daily; (c) ciprofloxacin $250 \mathrm{mg}$ twice daily.

Patients who could not tolerate any of these antibiotics received cefuroxime $250 \mathrm{mg}$ twice daily. The study was not blind as it was not placebo controlled.

The end point of the study was stent occlusion or death. Stent occlusion was defined as reappearance of jaundice with a serum bilirubin concentration of at least $50 \mu \mathrm{mol} / 1$, or episodes of cholangitis. In each

Characteristics of patients randomised to active treatment and no treatment groups. (Mean (SD) values are given except age for which median and range are given)

\begin{tabular}{lcc}
\hline & $\begin{array}{l}\text { Active } \\
\text { treatment } \\
(n=31)\end{array}$ & $\begin{array}{l}\text { No } \\
\text { treatment } \\
(n=39)\end{array}$ \\
\hline Median (range) age (y) & $68(64-85)$ & $70(62-82)$ \\
Bilirubin (SD) $\mu \mathrm{mol} / 1$ & $148(68)$ & $131(52)$ \\
Alkaline phosphatase (SD) $\mu / 1$ & $424(112)$ & $396(88)$ \\
Duration of jaundice before stent & $21(10)$ & $24(12)$ \\
$\quad(S D)$ days & 24 & 27 \\
Pancreatic carcinoma & $112(48)$ & $107(43)$ \\
Creatinine (SD) $\mu \mathrm{mol} / 1$ & & \\
\hline
\end{tabular}

Gastrointestinal Hospital, Edinburgh 23 March 1994
} 


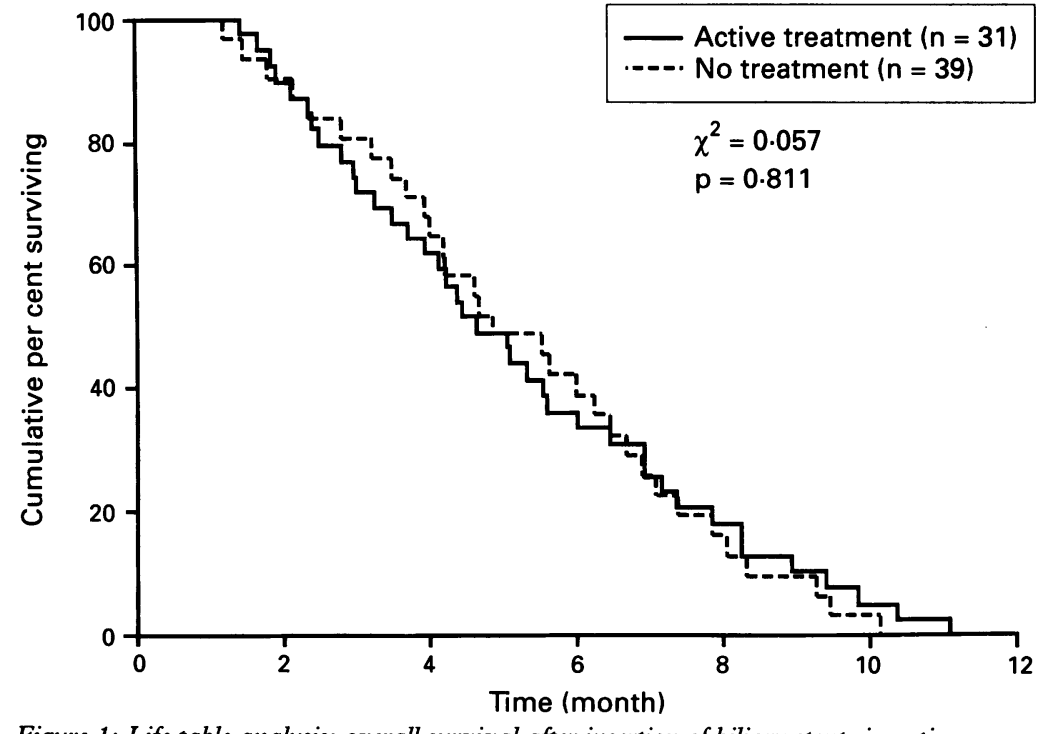

Figure 1: Life table analysis: overall survival after insertion of biliary stents in active treatment $\mathrm{v}$ no treatment groups. case suspected stent occlusion was confirmed by ERCP and examination of the removed stent. None of the patients who died showed features of stent occlusion before death.

Patients were followed up by SG and their general practitioners and none were lost to follow up. The follow up was complete and all patients were followed up until death or stent occlusion. The median follow up period was 32 weeks (range 5-48 weeks).

\section{STATISTICAL ANALYSIS}

Comparison of the two groups to check if they were well matched was done by the Student $t$ test for unpaired samples. The number of stent occlusions in the two groups was compared by the $\chi^{2}$ squared test with Yates's correction. Duration of survival and stent patency were compared by the Wilcoxon test for unpaired samples. Life table

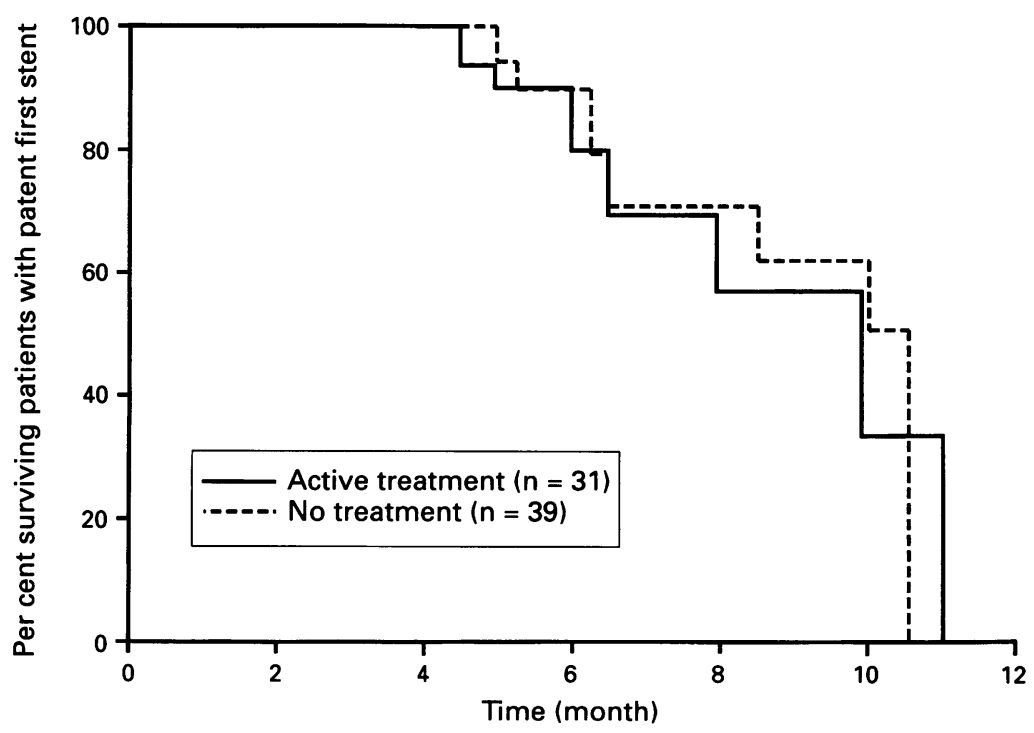

Figure 2: Comparison of stent patency: per cent surviving patients with patent first stent in active treatment and no treatment groups during the study period. analysis using Kaplan-Meier curves was used to compare survival percentage between the two groups.

\section{ETHICAL CONSIDERATIONS}

Ethical permission for the study was obtained from the Lothian committee for ethics of medicine and oncology. Each patient gave fully informed, written consent.

\section{Results}

Of the 87 patients considered for the study, 70 (40 men) were randomised. Thirty one were randomised to active treatment and 39 patients to supportive treatment. Fourteen patients were excluded because their prognosis was considered extremely poor and three others were excluded because stenting did not relieve jaundice.

The two groups were well matched with regards to age, presenting serum bilirubin concentration, duration of jaundice before stenting, and serum creatinine concentration (Table). After randomisation four patients in the active treatment group stopped antibiotic treatment after a 4-7 week treatment period because of side effects (drug rash in two patients, diarrhoea in one case, and nausea in a further patient). Two further patients received only two antibiotics (cefuroxime and ciprofloxacin) because of intolerance to metronidazole and ampicillin. These six patients were included in the active treatment group for analysis on an intention to treat basis. None occluded their stents.

Median survival in the active treatment group was 21 (range 5-44) weeks while median survival in the no treatment group was $20(6-48)$ weeks. There were seven episodes of stent occlusion in the active treatment group and eight episodes of stent occlusion in the no treatment group. The median time to stent occlusion was 26 weeks (range 18-44 weeks) in the active treatment group and 28 weeks (range 20-42 weeks) in the no treatment group. None of these parameters were significantly different.

In the active treatment group, four episodes of stent occlusion occurred in patients with $10 \mathrm{~F}$ stents (patent for $20,26,32,44$ weeks) and, three episodes of stent occlusion occurred in patients with patients with $12 \mathrm{~F}$ stents (patent for $18,24,40$ weeks). In the no treatment group, five episodes of stent occlusion occurred in patients with $10 \mathrm{~F}$ stents (patent for 20, 25, 26, 34, 42 weeks) and, the other three episodes occurred in patients with $12 \mathrm{~F}$ stents (patent for $21,30,40$ weeks). Eight of 15 blocked stents were $10 \mathrm{~cm}$ long, six of them were $8 \mathrm{~cm}$ long, and one $12 \mathrm{~cm}$ long.

The life table analysis for the two groups (Fig 1) did not show any difference between the cumulative per cent survival $(p=0 \cdot 8)$. Figure 2 shows the percentage of surviving patients at any point in the study period having a patent first stent; there were no differences between the two groups of patients. 


\section{Discussion}

This open randomised controlled trial showed no benefit for cyclical antibiotics and ursodeoxycholic acid either in terms of patient survival or duration of stent patency. Only a few stents occluded and only $18 \%$ of the 70 randomised patients required readmission for restenting. A previous trial of aspirin and doxycycline had also shown that intervention failed to influence the incidence of stent occlusion. ${ }^{4}$

The time to stent occlusion reported in this study was slightly longer than the usually quoted figure of three months, ${ }^{5}$ although similar stent patency rates have been reported by some groups. ${ }^{67}$ Our exclusion of proximal or bifurcation bile duct tumours from this study may partly explain this. Most patients died with their first stent in situ and this has economic relevance to the use of metal stents. Expandable metal stents have significantly lower occlusion rates ${ }^{8}$ but expense precludes their use in many poorly funded British units. Consequently, these stents can only be justified for units undertaking a large number of stenting procedures where the increased financial outlay of metal stents is balanced by a lower rate of readmission to hospital for restenting. The comparatively low readmission rate for stent occlusion in this study suggests that only very active units can justify a universal policy of metal stent usage.

The comparatively small number of patients in this study and the low occlusion rates make it difficult to exclude a type II error. The power of this study is 0.8 to detect a $75 \%$ difference in stent occlusion rate at $p=0.05$ value. At least 219 patients would be necessary to detect a $50 \%$ reduction in stent occlusion rate with the same power; to detect a smaller reduction of $25 \%$, a sample size of at least 945 patients would be required. Such a study would be difficult to complete in one centre and the clinical value of the finding would be extremely limited, particularly when the morbidity and expense of active treatment are balanced against a modest difference in stent blockage rates. As a large proportion of patients die before their stents occlude, enormous numbers of patients have to be treated before any benefit would become obvious even with an effective treatment.

This study may be criticised on the basis of the choice and timing of active treatment. Ampicillin, although well excreted in bile, may not be very effective prophylaxis against the enteral bacteria that commonly colonise the biliary tree after stenting. Furthermore, patients were not randomised until two weeks after stenting and it is possible that bacterial adhesion could have started within this period. Biofilm matrix may offer the bacteria a survival advantage against even high tissue concentrations of antibiotics. ${ }^{9}$ All patients, however, had received prophylactic antibiotics before ERCP and they received IV piperacillin for 48 hours after stent insertion. This is likely to have inhibited early bacterial biofilm formation. Ursodeoxycholic acid, although well tolerated, is only a modest choleretic agent and a more potent agent such as ursocholic acid might have been more effective. The generally recommended dose of $10 \mathrm{mg} / \mathrm{kg} /$ day was used $^{10}$ and a single night time dose has been used in cholestatic diseases. ${ }^{11}$ Higher doses (15 mg/kg/day), however, may produce more choleresis and may be worth investigating in future trials. It has to be noted that apart from the well recognised effect of choleresis, ${ }^{12}$ ursodeoxycholic acid has other important actions such as change of the composition of bile and the immune response, which potentially may favourably influence biliary sludging. ${ }^{3}$

Thus, further studies using other potentially valuable prophylactic regimens seem worthwhile. Only longterm survivors are likely to benefit but, as yet, there are no reliable ways of predicting longterm survival in this group of patients.

This study was presented as a poster at the 1993 spring meeting of the British Society of Gastroenterology at Manchester and was published as an abstract (Gut 1993; 34 (suppl 1): S69). This study was also presented as a poster in the biliary disorders section of the Annual American Gastroenterology Association meeting in Boston 1993 (Gastroenterology 1993; 104: A361).

1 Speer AG, Cotton PB, Rode J, Seddon AM, Neal CR, Holton J, et al. Biliary stent blockage with bacterial biofilm: a light and electron microscopic study. Ann Intern Med 1988; 108: 546-53.

2 Leung JWC, Ling TKW, Kung JLS, Vallance-Owen J. The role of bacteria in the blockage of biliary stents. role of bacteria in the blockag

3 de Caestecker JS, Jazrawi RP, Petroni ML, Northfield TC. Ursodeoxycholic acid in chronic liver disease. Gut 1991; 32: 1061-5.

4 Smit JM, Out MMJ, Groen AK. A placebo controlled study on the efficacy of aspirin and doxycycline in preventing clogging of biliary endoprosthesis. Gastrointest Endosc 1985; 35: 485-9.

5 Speer AG, Cotton PB, Macrae KD. Endoscopic management of malignant biliary tract obstruction: stents of $10 \mathrm{~F}$ gauge are preferable to stents of $8 \mathrm{~F}$ gauge. Gastrointest Endosc 1988; 34: 412-7.

6 Frakes JT, Johanson JF, Stake JJ. Optimal timing for stent replacement in malignant biliary tract obstruction. replacement in malignant biliary

7 Matsuda Y, Shimakura K, Akamatsu T. Factors affecting the patency of stents in malignant biliary obstructive disease: univariate and multivariate analysis. $A m \mathcal{F}$ Gastroenterol 1991; 86: 843-9.

8 Knyrim K, Wagner HJ, Pausch J, Vakil N. A prospective randomized controlled trial of metal stents for malignant obstruction of the common bile duct. Endoscopy 1993; 25: 207-12.

9 Costerton JW, Cheng KJ, Geesey GG. Bacterial biofilm in nature and disease. Annu Rev Microbiol 1987; 41: 435-64.

10 Ward A, Brogden RN, Heel RC, Speight TM, Avery GS. Ursodeoxycholic acid. A review of its pharmacological properties and therapeutic efficacy. Drugs 1984; 27: properties

11 Jazrawi RP, de Caestecker JS, Goggin PM, Britten AJ, Joseph AEA, Maxwell JD, et al. Kinetics of hepatic bile acid handling in cholestatic liver disease: effect of 12 Orsodeoxycholic acid. Gastroenterology 1994; 106: 134-42. acid and canrenoate induced biliary bicarbonate secretion and the effect on glucose and amino acid induced cholestasis. Scand $\mathcal{F}$ Gastroenterol 1991; 26: 513-22. 